\title{
Molecular cloud structure and star formation near HH 216 in M 16
}

\author{
M. Andersen ${ }^{1, \star}$, J. Knude ${ }^{2}$, B. Reipurth ${ }^{3}$, A. Castets ${ }^{4}$, L. Å. Nyman ${ }^{5,6}$, M. J. McCaughrean ${ }^{1}$, and S. Heathcote ${ }^{7}$ \\ 1 Astrophysikalisches Institut Potsdam, An der Sternwarte 16, 14482 Potsdam, Germany \\ 2 Niels Bohr Institute for Astronomy, Physics and Geophysics, 2100 Copenhagen, Denmark \\ 3 Institute for Astronomy, University of Hawaii, 2680 Woodlawn Drive, Honolulu, HI 96822, USA \\ ${ }^{4}$ Observatoire de l'Université de Bordeaux I, BP 89, 33270 Floirac, France \\ 5 SEST, ESO-La Silla, Casilla 19001, Santiago 19, Chile \\ ${ }^{6}$ Onsala Space Observatory, 43992 Sweden \\ 7 SOAR, Cerro Tololo Inter-American Observatory, Casilla 603, La Serena, Chile
}

Received 30 October 2001 / Accepted 25 September 2003

\begin{abstract}
We present millimetre, optical, and near-infrared observations of M 16 in the vicinity of the Herbig-Haro object HH 216. The line profiles of the CO $(J=2-1)$ spectra are broad and consist of both emission originating from four warm molecular cores and a large cloud or cloud system across the whole field. The CS $(J=2-1)$ and the $\mathrm{C}^{18} \mathrm{O}(J=2-1)$ lines are relatively broad compared with those observed in low-mass star formation regions, but not unusually broad for highermass star-forming regions. The virial masses found are much larger than the mass estimates based on the assumption of LTE. The optical images suggest that HH 216 is the terminating bow shock of a large Herbig-Haro flow which includes a jet. A possible location for the driving source is suggested, from the presence of a very reddened point source associated with what may be a small reflection nebula at the tip of a dense molecular "trunk". Another reflection nebula associated with the same core is also detected. This demonstrates that star formation is taking place in the trunk.
\end{abstract}

Key words. stars: formation - ISM: H II regions - ISM: jets and outflows - ISM: molecules - ISM individual object: M 16 ISM individual object: HH 216

\section{Introduction}

The interplay between UV flux from newly-born early-type stars and adjacent molecular clouds has a profound influence on the structure of the clouds. The UV flux will evaporate the outer layers of the cloud and compress the remaining gas. Parts of the molecular cloud can be left behind, shaped as cometary globules or elephant trunks, both of which have been proposed as active sites of triggered star formation.

Probing the physical parameters in elephant trunks should provide the initial conditions for triggered star formation.

The H II region M 16, also known as the Eagle Nebula, is located close to the galactic plane $(l, b)=\left(17^{\circ}, 1^{\circ}\right)$ and is associated with the star cluster NGC 6611 . The cluster has been subjected to intense study in order to determine both its distance and stellar content (e.g. Hillenbrand et al. 1993, hereafter Hi93; de Winter et al. 1997; Belikov et al. 2000). The distance to $\mathrm{M} 16$ is $2.0 \pm 0.1 \mathrm{kpc}$ according to $\mathrm{Hi} 93$ and we assume that distance here, although a distance of $2.6 \mathrm{kpc}$ has also been suggested (Belikov et al. 2000).

Send offprint requests to: M. Andersen, e-mail: mortena@aip.de

* Work conducted at The Niels Bohr Institute for Astronomy Physics and Geophysics, Astronomical Observatory.
The $\mathrm{H}$ II region is created by numerous $\mathrm{O}$ and $\mathrm{B}$ stars within NGC 6611. The age of the cluster, and therefore the H II region, was estimated by Hi93 to be a few Myr, although the presence of an evolved $\mathrm{O}$ star indicates that some star formation was taking place as long as $6 \mathrm{Myr}$ ago. At the same time, pre-main sequence stars with ages around $1 \mathrm{Myr}$ are found within the cluster.

The OB stars in M 16 have sculpted a number of elephant trunks, well-known from the HST imaging of Hester et al. (1996, hereafter He96). The top of the main group of elephant trunk structures has been observed in the millimetre by Pound (1998, hereafter P98) and White et al. (1999, hereafter Wh99). It was found that most of the mass in the trunks is located in their tips. Since the external pressure was observed to be larger than the internal pressure, Wh99 proposed that the tip of one of the trunks could actually be a very young pre-Class 0 object. Recent near-infrared observations by Sugitani et al. (2002), Thompson et al. (2002) and McCaughrean \& Andersen (2002) show massive stars forming at the tip of both this and the middle trunk, not consistent with the pre-Class 0 claim.

Another sign of present-day star formation in the region was discovered by Meaburn \& White (1982) in the form of a Herbig-Haro object (HH216, originally known as M16-HH1) 
located south-east of the part of the elephant trunks studied by He96. Early studies in the near-infrared found no convincing candidate for the driving source (Krautter et al. 1984), although these observations did not go very deep. Walsh \& White (1982) found several infrared sources within M 16, one situated $40^{\prime \prime}$ north-west of $\mathrm{HH} 216$. This is not likely to be the source since higher resolution observations have shown this to be a quadruple of bright, but not very red stars and these are not associated with any molecular material as seen in the images of, for example, McCaughrean \& Andersen (2002).

Only the tips of the trunks have previously been investigated in detail in millimetre studies. The regions further south are also interesting for several reasons. What are the physical parameters in the region partly shielded by the tip of the pillars? Are the conditions such that star formation might take place in this region? An $\mathrm{HH}$ object is present, but no driving source has been found; is it located deep within molecular material?

In order to study these issues, multi-wavelength observations of the southern part of the elephant trunks have been obtained. Millimetre observations of the rotational transitions ${ }^{12} \mathrm{C}^{16} \mathrm{O}(J=2-1),{ }^{12} \mathrm{C}^{18} \mathrm{O}(J=2-1)$ and ${ }^{12} \mathrm{C}^{32} \mathrm{~S}(J=2-1)$, optical [S II], $\mathrm{H} \alpha$ and red continuum images, and near-infrared $J_{\mathrm{s}}, H, K_{\mathrm{s}}$ images have been obtained. The near-infrared data have briefly been discussed by McCaughrean \& Andersen (2002), and a more detailed discussion on the data reduction will be given in Andersen et al. (in prep.).

\section{Observations and reduction}

\subsection{Millimetre data}

The millimetre data were obtained with the SEST on La Silla on July 12-15 1998. The observations were aimed at detecting the rotational transitions of three molecular tracers: $\mathrm{CO}(2-1)$ at $230.5 \mathrm{GHz}, \mathrm{C}^{18} \mathrm{O}(2-1)$ at $219.5 \mathrm{GHz}$ and $\mathrm{CS}(2-1)$ at $98.0 \mathrm{GHz}$. For brevity, the three molecular transitions are referred to as $\mathrm{CO}, \mathrm{C}^{18} \mathrm{O}$, and $\mathrm{CS}$, respectively. Frequency switching was used in all the observations with a frequency throw corresponding to $40 \mathrm{~km} \mathrm{~s}^{-1}$. An important consequence of the decision to use frequency switching is that emission from high velocity outflows will be hard to detect. The integration time for each spectrum was $120 \mathrm{~s}$. The velocity resolution was $0.054 \mathrm{~km} \mathrm{~s}^{-1}, 0.057 \mathrm{~km} \mathrm{~s}^{-1}$ and $0.128 \mathrm{~km} \mathrm{~s}^{-1}$ for $\mathrm{CO}, \mathrm{C}^{18} \mathrm{O}$, and $\mathrm{CS}$, respectively. The pointing was checked regularly using $\mathrm{SiO}$ maser sources and was found to be accurate to $3^{\prime \prime} \mathrm{rms}$, which is the pointing accuracy of the SEST. The $\mathrm{C}^{18} \mathrm{O}$ and $\mathrm{CS}$ observations were obtained simultaneously. The millimetre data was reduced with the CLASS and GRAPHIC packages at the Laboratoire d'Astrophysique de Grenoble.

The system temperature for $\mathrm{CO}$ and $\mathrm{CS}$ observations was fairly stable, 440-600 K for $\mathrm{CO}$ and $240-310 \mathrm{~K}$ for $\mathrm{CS}$, whereas the atmospheric conditions changed during the observations, resulting in a system temperature varying between $240 \mathrm{~K}$ and $700 \mathrm{~K}$ for $\mathrm{C}^{18} \mathrm{O}$. The FWHP beam widths are $24^{\prime \prime}$ for the $\mathrm{CO}$ and the $\mathrm{C}^{18} \mathrm{O}$ observations and $51^{\prime \prime}$ for the $\mathrm{CS}$ observations. The $\mathrm{CO}$ and $\mathrm{CS}$ spectra were obtained at half beamwidth sampling, while the $\mathrm{C}^{18} \mathrm{O}$ spectra were obtained at full beam-width sampling. The intensities have been converted into the main beam brightness temperature scale by adopting a main beam brightness efficiency of $0.46,0.47$, and 0.75 for the $\mathrm{CO}, \mathrm{C}^{18} \mathrm{O}$, and $\mathrm{CS}$ data, respectively. A second order polynomial baseline has been applied to the $\mathrm{CO}$ and $\mathrm{CS}$ spectra, and a third order to the $\mathrm{C}^{18} \mathrm{O}$ spectra. The rms noise in one channel was $0.3 \mathrm{~K}, 0.1-0.2 \mathrm{~K}$, and $0.1 \mathrm{~K}$ for $\mathrm{CO}, \mathrm{C}^{18} \mathrm{O}$, and $\mathrm{CS}$, respectively, the variation in the noise on the $\mathrm{C}^{18} \mathrm{O}$ data reflects the highly varying system temperature. The region observed has some overlap with the region observed by Wh99.

\subsection{Optical and IR data}

$\mathrm{H} \alpha$ and [S II] $\lambda \lambda 6716,6731 \AA$ images were obtained with the $4 \mathrm{~m}$ telescope at Cerro Tololo on August 51999 in 1'.5 seeing. Each image was a 3 min integration.

Additional $\mathrm{H} \alpha$, [S II] $\lambda \lambda 6716,6731 \AA$, and red continuum $\left(\lambda_{\text {cen }}=6838 \AA\right)$ frames were obtained with the $1.54 \mathrm{~m}$ Danish telescope at La Silla on April 26, 2001.

The total integration time was $9 \mathrm{~min}$ in each filter. The seeing was 0.8 during the observations and the pixel scale is 0.'395/pixel. Standard bias subtraction and flat-field reduction has been applied to both the CCD data sets.

The $J_{\mathrm{s}}, H$ and $K_{\mathrm{s}}$ band frames were obtained on April 10 and May 8-10 2001 with the ISAAC instrument mounted on VLT UT1 ANTU (ESO program 67.C-0595). The integration times for the region discussed here were $50 \mathrm{~s}$ for $H$, and $K_{\mathrm{s}}$, respectively and 200 seconds for $J_{\mathrm{s}}$. The pixel scale was $0.147 /$ pixel and the seeing was $0.38,0.36$, and $0.33^{\prime \prime}$ FWHM in $J_{\mathrm{s}}, H$, and $K_{\mathrm{s}}$, respectively.

\section{Observational results}

\subsection{The $\mathrm{C}^{18} \mathrm{O}$ and $\mathrm{CS}$ data}

The slightly smoothed, integrated intensity map of the $\mathrm{C}^{18} \mathrm{O}$ emission is shown in Fig. 1. Several regions have conspicuous emission and can be considered as molecular cores due to their sizes. The same cores can be identified in the CS integrated map, but less readily since the CS beam is twice as large. No structure is evident in the CS map that is not visible in the $\mathrm{C}^{18} \mathrm{O}$ map. For direct comparison the integrated CS map is shown in Fig. 2. Figure 3 shows the $\mathrm{C}^{18} \mathrm{O}$ velocity map in bins of $0.75 \mathrm{~km} \mathrm{~s}^{-1}$.

We define a core as an intensity peak standing clearly out from its surroundings relative to the noise in Figs. 1 and 3. This effectively means an intensity peak above $1.5 \mathrm{~K} \mathrm{~km} \mathrm{~s}^{-1}$ in Fig. 1. Four cores are identified in Fig. 1 and listed in Table 1. Notice that core $S$ is at the edge of the map and therefore is not well defined. It could be larger than is observed here, extending to the west. We therefore only list the coordinates for core $\mathrm{S}$ but do not try to derive a mass for it, given the uncertainty in determining its size. Besides the cores, emission can be seen extending from core $\mathrm{C}$ towards the $\mathrm{SSE}$, and probably beyond the observed region. We refer to this feature as a "finger" in the following. The term finger is used instead of elephant trunk to avoid confusion with the elephant trunks further to the north observed by He96, P98 and Wh99. A possible fifth core can be seen between core $\mathrm{S}$ and core $\mathrm{C}$ at the velocities $22.5 \mathrm{~km} \mathrm{~s}^{-1}$ 


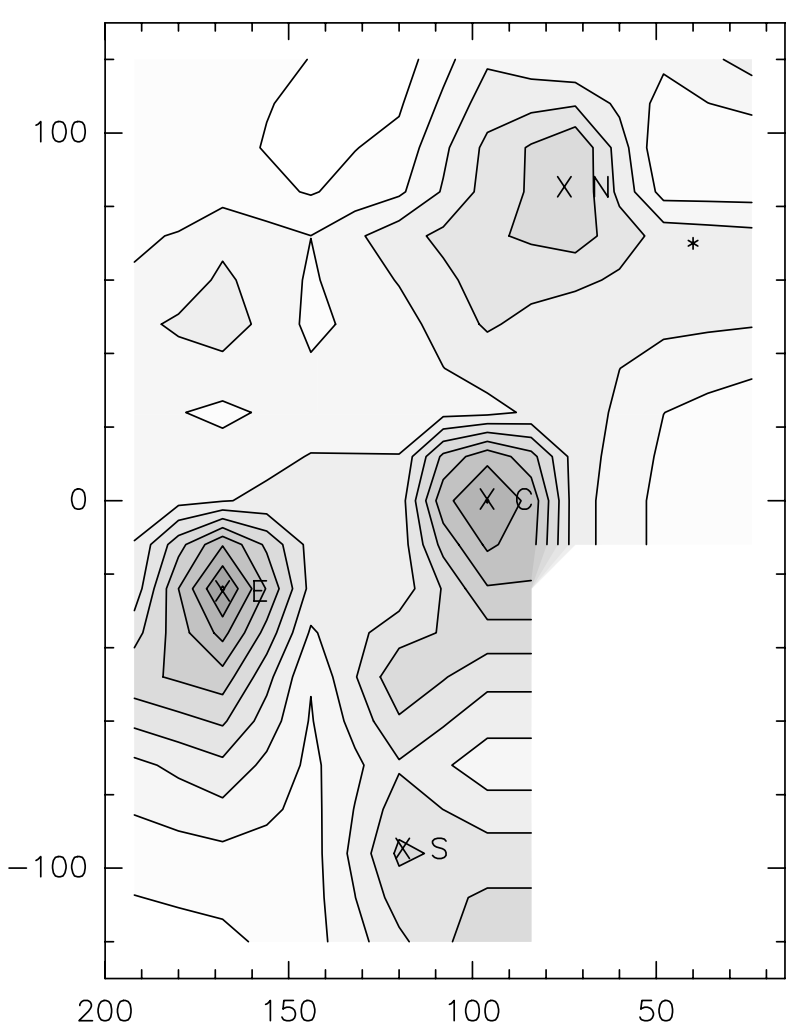

Fig. 1. The integrated $\mathrm{C}^{18} \mathrm{O}$ emission. Coordinate offsets are in arcseconds from the reference position $\left(2000: 18^{\mathrm{h}} 18^{\mathrm{m}} 52.8^{\mathrm{s}},-13^{\circ} 53^{\prime} 00^{\prime \prime}\right)$ The emission is integrated from $15-35 \mathrm{~km} \mathrm{~s}^{-1}$. The contours start at $0 \mathrm{~K} \mathrm{~km} \mathrm{~s}^{-1}$ and the increment is $0.5 \mathrm{~K} \mathrm{~km} \mathrm{~s}^{-1}$. Four cores and an elephant trunk are evident. The cores are marked with letters according to their name given in Table 1 and their centre are marked with an $x$ and their corresponding letter. The HH object HH 216 is marked with an asterix.

Table 1. The position and effective radius of the four cores identified in $\mathrm{C}^{18} \mathrm{O}$. Core $\mathrm{S}$ is smaller than the beam-width and is therefore seen as a point source.

\begin{tabular}{llll}
\hline \hline Core & RA $(2000)$ & Dec $(2000)$ & radius \\
\hline North $(\mathrm{N})$ & $18^{\mathrm{h}} 18^{\mathrm{m}} 58.25^{\mathrm{s}}$ & $-13^{\circ} 51^{\prime} 45^{\prime \prime}$ & $25^{\prime \prime}$ \\
Centre (C) & $18^{\mathrm{h}} 18^{\mathrm{m}} 59.25^{\mathrm{s}}$ & $-13^{\circ} 53^{\prime} 00^{\prime \prime}$ & $25^{\prime \prime}$ \\
East (E) & $18^{\mathrm{h}} 19^{\mathrm{m}} 04.25^{\mathrm{s}}$ & $-13^{\circ} 53^{\prime} 25^{\prime \prime}$ & $30^{\prime \prime}$ \\
South (S) & $18^{\mathrm{h}} 19^{\mathrm{m}} 00.75^{\mathrm{s}}$ & $-13^{\circ} 54^{\prime} 45^{\prime \prime}$ & $12^{\prime \prime}$ \\
\hline
\end{tabular}

and $23.25 \mathrm{~km} \mathrm{~s}^{-1}$, although we consider this emission to be part of the finger. All the cores are recognized at the 3 sigma level in the velocity map Fig. 3, even the possible core $\mathrm{S}$ is present at $22.5-23.25 \mathrm{~km} \mathrm{~s}^{-1}$

The radii given in Table 1 correspond to the half widthhalf-intensity contour. The half-intensity core radius was found either as the radius at the half-peak intensity contour or, in case of a non-circular core, as the square root of the product of half the minor and half the major axes of the half-peak intensity contour. All the cores have radii comparable to the beam-width in $\mathrm{C}^{18} \mathrm{O}$ and a full extension almost equivalent to the CS beamwidth. Radius estimates are therefore based on the $\mathrm{C}^{18} \mathrm{O}$ map.

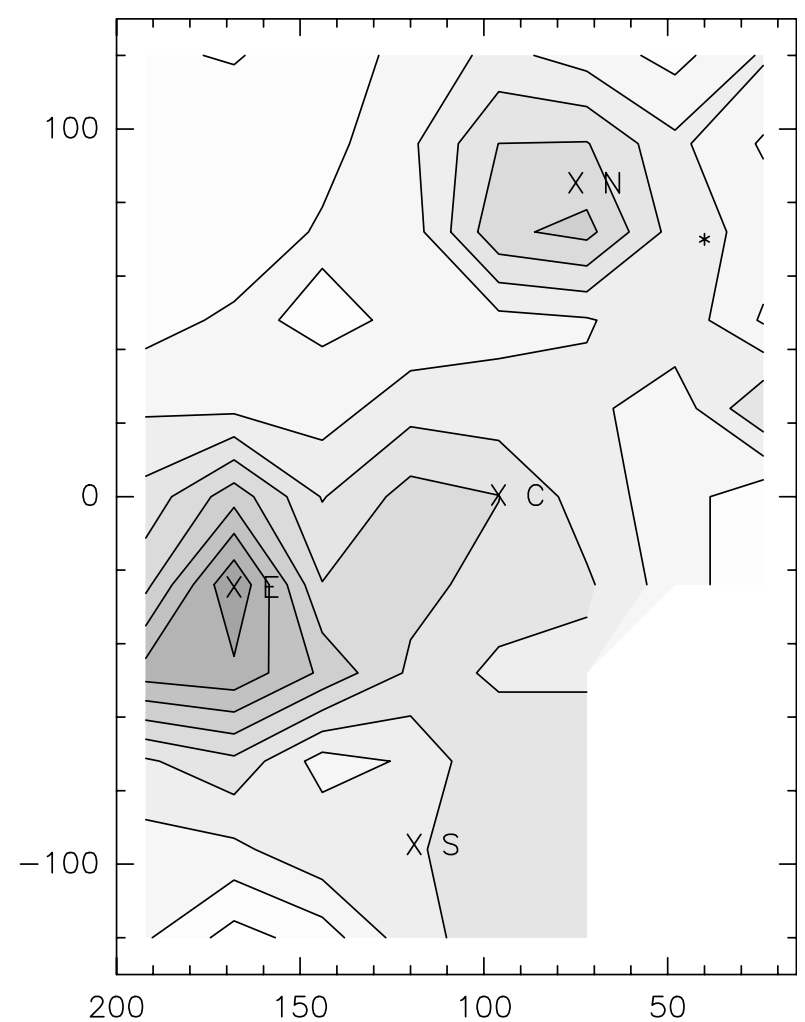

Fig. 2. The integrated CS emission. The emission is integrated from $15-35 \mathrm{~km} \mathrm{~s}^{-1}$. The contours start at $0 \mathrm{~K} \mathrm{~km} \mathrm{~s}^{-1}$ and the increment is $0.5 \mathrm{~K} \mathrm{~km} \mathrm{~s}^{-1}$. The centre of the cores are marked with an $\times$ and their corresponding letter. The HH object HH 216 is marked with an asterix.

The lines observed in $\mathrm{C}^{18} \mathrm{O}$ and $\mathrm{CS}$ in general have fullwidth half-maximum of more than $1.5 \mathrm{~km} \mathrm{~s}^{-1}$, higher than usually seen in quiescent cores (e.g. Cernicharo 1991), but not unusual for more massive star forming regions. The line widths observed here are comparable with the widths found further north in the elephant trunks by Wh99. The velocity map of the $\mathrm{CS}$ emission is very similar to the $\mathrm{C}^{18} \mathrm{O}$ map but with lower spatial resolution. What is perhaps a bit surprising is that the widths of the $\mathrm{C}^{18} \mathrm{O}$ and $\mathrm{CS}$ spectra are similar at a given spatial position. In at least two of the elephant trunks, different widths have been measured for the 3-2 transitions of the same molecules (Wh99).

From Fig. 3, it appears that the only structure in the map with a velocity gradient is core $\mathrm{E}$. This core could be rotating with the northern part moving towards us and the southern part away from us. The finger apparently has no velocity gradient at all within the velocity resolution obtained in this study. The lack of a velocity gradient is at odds with a scenario where the finger has been formed through a Rayleigh-Taylor instability, and is in agreement with the findings for the elephant trunks by P98. However, given that we only see one velocity component, it is hard to distinguish between the different formation scenarios even with the velocity information, and a Rayleigh-Taylor instability can therefore not be ruled out. 


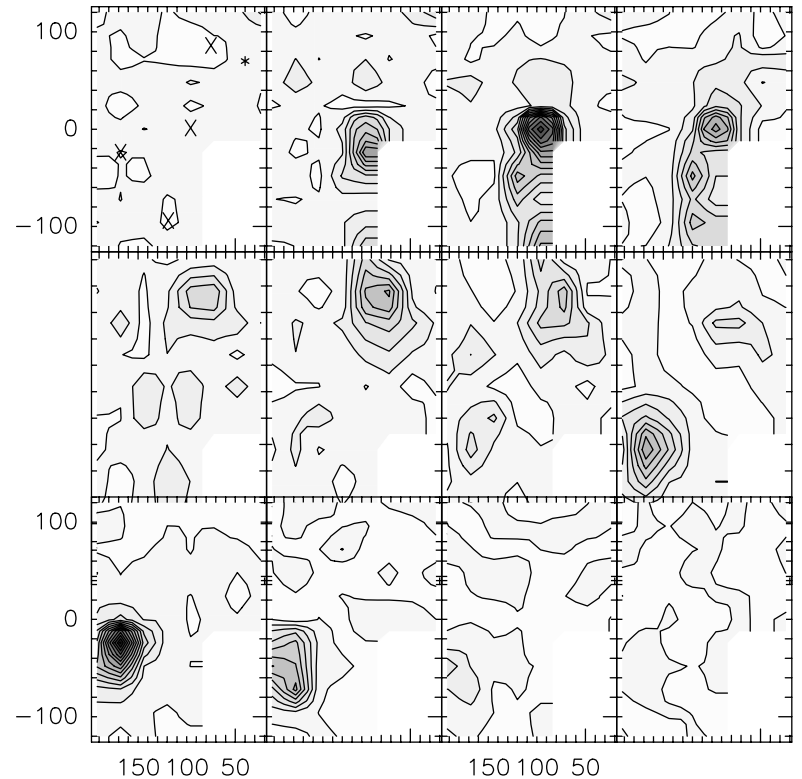

Fig. 3. The $\mathrm{C}^{18} \mathrm{O}$ emission in velocity bins of $0.75 \mathrm{~km} \mathrm{~s}^{-1}$ starting at $21 \mathrm{~km} \mathrm{~s}^{-1}$ and ending at $29.25 \mathrm{~km} \mathrm{~s}^{-1}$. The lowest contour is $-0.2 \mathrm{~K} \mathrm{~km} \mathrm{~s}^{-1}$ and the increment is $0.2 \mathrm{~K} \mathrm{~km} \mathrm{~s}^{-1}$. In the first panel each core is indicated with an $\times$ and $\mathrm{HH} 216$ with an asterix.

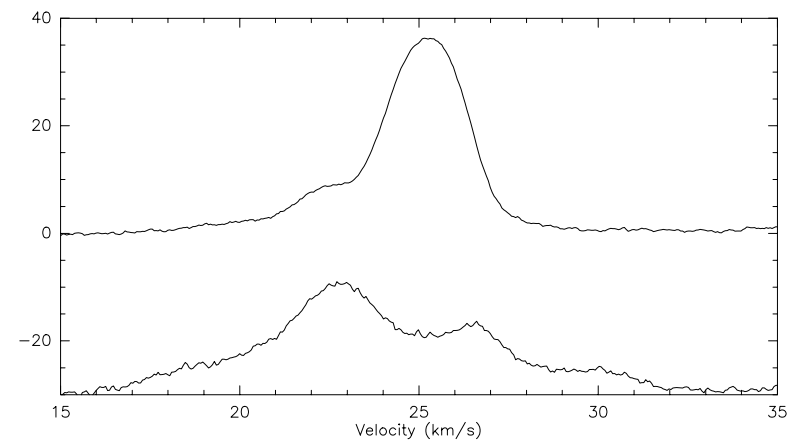

Fig. 4. Comparison of a $\mathrm{CO}$ spectrum associated with the core N (top spectrum) and a $\mathrm{CO}$ spectrum for a location not associated with a core (bottom spectrum). The scale is main beam brightness temperature, but the lower spectrum has been offset by $-25 \mathrm{~K}$ and has been multiplied by a factor of two.

\subsection{The CO data}

Besides the $\mathrm{CO}$ emission associated with the cores that are also seen in $\mathrm{CS}$ and $\mathrm{C}^{18} \mathrm{O}$, there is a broad component present in all the CO spectra. A close examination of the CO (3-2) spectra presented by Wh99 also shows this component, although it appears fainter than in this region. It is only present in the southern part of the region observed in CO by Wh99. The FWHM of this broad component is roughly $6-8 \mathrm{~km} \mathrm{~s}^{-1}$, varying from spectrum to spectrum. The broad velocity component is apparently composed of several narrower components. An example of a $\mathrm{CO}$ spectrum associated with a core and a $\mathrm{CO}$ spectrum from a position away from any core, showing the broad component, can be seen in Fig. 4.

The complex shape could be due to self-absorption, such that the line-profile is severely distorted. However, since for many of the $\mathrm{CO}$ spectra showing this complicated shape no

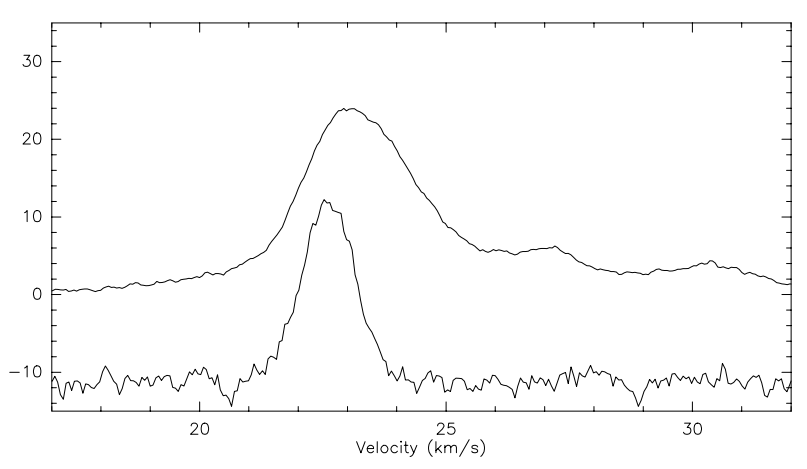

Fig. 5. Comparison of the $\mathrm{C}^{18} \mathrm{O}$ emission (lower spectrum) and $\mathrm{CO}$ emission associated with core $\mathrm{C}$. The peak of the core is offset $12^{\prime \prime}$ from this position in both RA and DEC. No $\mathrm{C}^{18} \mathrm{O}$ spectrum is available at the centre position since the spacing is coarser than for the $\mathrm{CO}$ spectra. The scale is main beam brightness temperature, but the $\mathrm{C}^{18} \mathrm{O}$ spectrum has been offset and enhanced relative to the $\mathrm{CO}$ spectrum. The continuum is at $0 \mathrm{~K}$ and the peak intensity at $3 \mathrm{~K}$. Notice the two spectra peaks at two different velocities.

$\mathrm{C}^{18} \mathrm{O}$ emission above the noise is present, the lines are probably not self-absorbed. The $\mathrm{C}^{18} \mathrm{O}$ peak position is in this case blueshifted relative to the $\mathrm{CO}$ peak, indicating some cooler material in front of the core.

Given that the broad component is present across the whole observed field and possibly a bit further north towards the elephant trunks, it is probably a remnant of the giant molecular cloud (GMC) out of which NGC 6611 was formed. Closer to the tip of the northern elephant trunks, the impact of the $\mathrm{UV}$ radiation is stronger and will presumably have dispersed much more of the GMC explaining why it is not seen closer to NGC 6611.

\subsection{Optical and near-infrared data}

Figure 6 directly compares the optical and near-infrared observations with each other. Core $\mathrm{C}$ and the finger, as identified in the millimetre map, can also be seen in the centre of Fig. 6 as a large windswept structure. The geometry of the finger, and the presence of bright rims surrounding it, shows that it is pointing directly towards the ionising OB stars.

The HH 216 object discovered by Meaburn \& White (1982) can be seen to the northwest, right beside core $\mathrm{N}$ at $(40,70)$ in Fig. 6. At the tip of the finger, several emission regions can be seen. Also, a string of emission features along the finger are seen, see also Fig. 7. In the [S II] image, they appear to terminate in a shock at the middle of the finger at $(130,-60)$. This is seen in both emission lines but not in the continuum, suggesting that it may be an $\mathrm{HH}$ object. This terminus of emission and $\mathrm{HH} 216$ are located symmetrically on either side of core C.

A long-slit spectrum has been obtained of $\mathrm{HH} 216$ and the emission knots in order to measure their radial velocities. High blue-shifted radial velocities of the order of $-150 \mathrm{~km} \mathrm{~s}^{-1}$ were found along the southern emission knots. HH 216, on the other hand, shows redshifted motion by the same amount. In the $\mathrm{K}$-band image shown in Fig. 6, a bright reflection nebula is seen almost at the centre of core $\mathrm{C}$. A relatively faint point source with some nebulosity around it is located further to the 


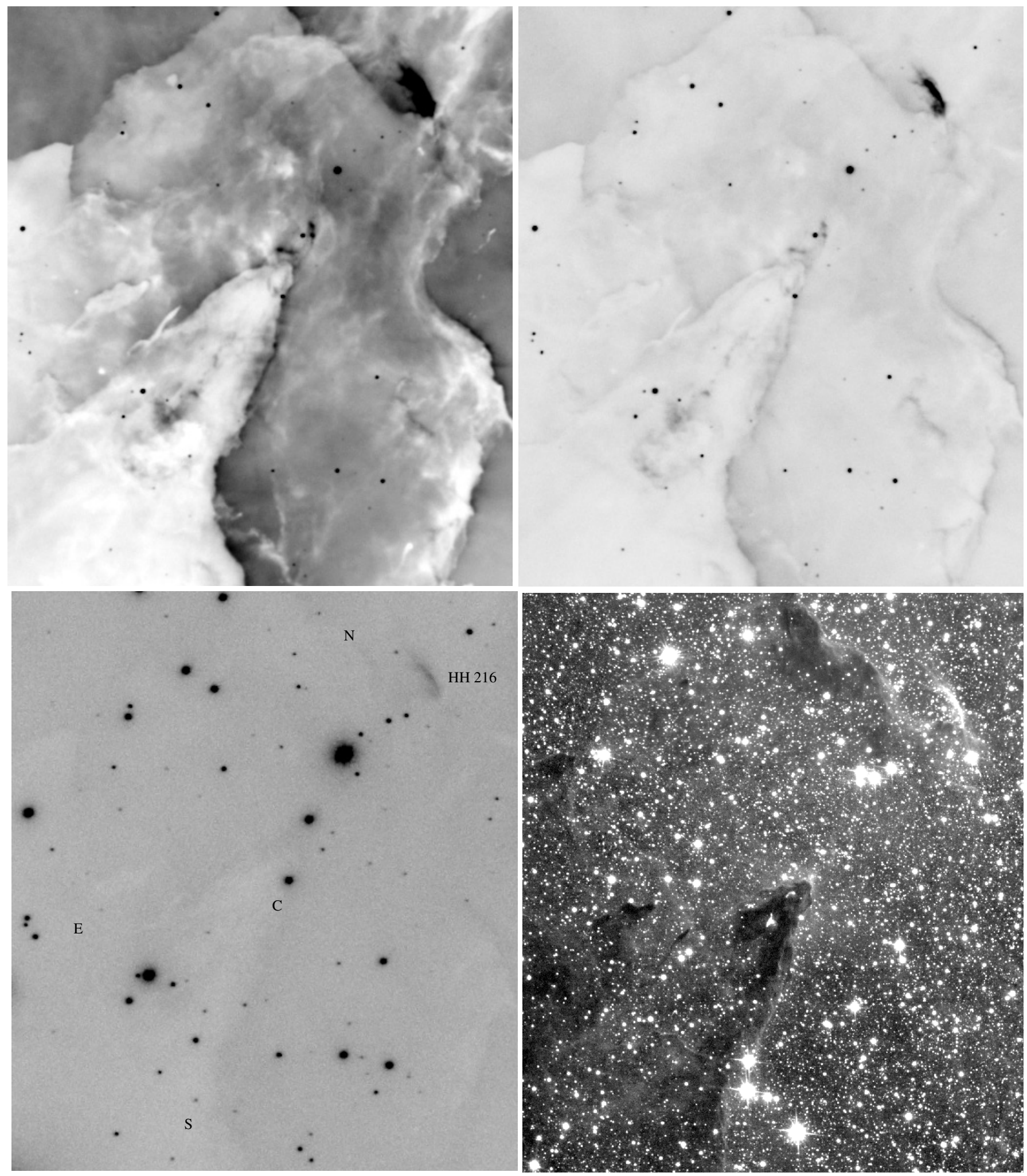

Fig. 6. A comparison of $\mathrm{H} \alpha$ (upper left), [S II] (upper right), continuum (lower left), and the $K_{\mathrm{s}}$ band emission (lower right). The regions shown are slightly smaller than the region in Fig. 9 (174" $\times 200^{\prime \prime}$, centred on the extended emission region). The intensity scale for H $\alpha$ and [S II] images are chosen to normalise stellar brightness. In the continuum image, cores and HH 216 are marked with letters next to them. Note the two very red extended objects associated with core C. As discussed in the text, one of them is probably driving by the driving source of $\mathrm{HH} 216$ and the candidate jet. The other can be the signpost of another star forming. Notice also how translucent the trunk is in the $K_{\mathrm{s}}$ band image compared to the emission line images. The finger is much narrower but core $\mathrm{C}$ is comparable in size to that measured at optical wavelengths. For a comparison with the molecular cores, see Fig. 9. 


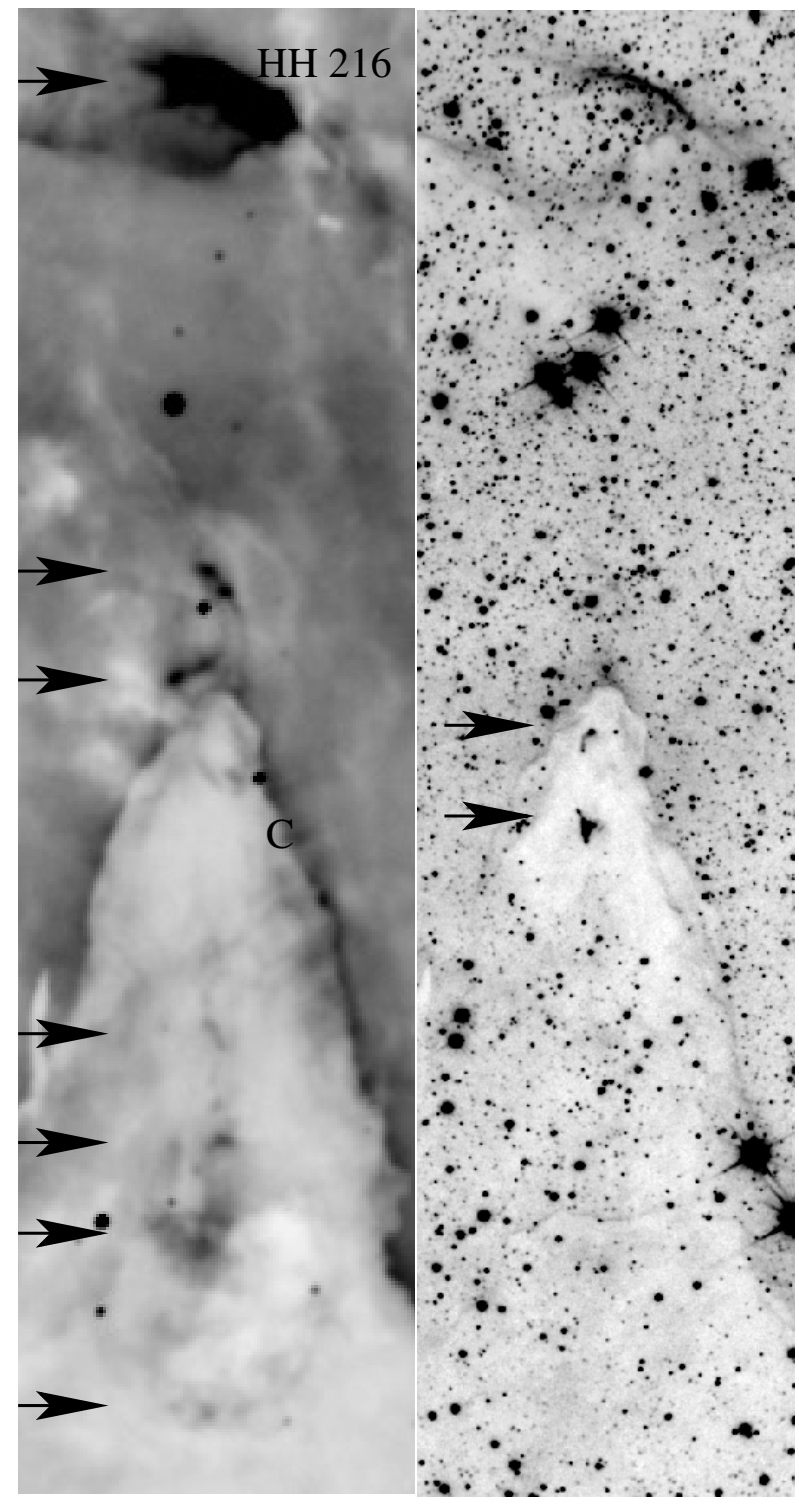

Fig. 7. Left: $\mathrm{H} \alpha$ image of the region of $\mathrm{HH} 216$ and other candidate $\mathrm{HH}$ objects. The arrows point to emission features that are possible $\mathrm{HH}$ objects and are seen both in this image and the [S II] image. Right: the $K_{\mathrm{s}}$ band image of the same region. Both images are rotated $45^{\circ}$. Arrows mark the nebulosity that we associate with the driving source (lower arrow) and the thin emission feature (upper arrow). Core $\mathrm{C}$ has been marked to the west of it and HH 216 is indicated with its name as well.

NW but still associated with core C. As discussed in Sect. 4.2, the southern emission knots are likely to be a counter HH flow to HH 216 and the driving source of the whole $\mathrm{HH}$ flow is presumably the faint point source to the $\mathrm{NW}$ in core C. Figure 8 shows the optical images also shown in Fig. 6 as a true-colour image and the $J_{\mathrm{s}}, H$ and $K_{\mathrm{s}}$ images also as a true-colour image. It is apparent from the near-infrared image that the two sources discussed above are indeed very red.

Figure 9 compares the [S II] emission with the $\mathrm{C}^{18} \mathrm{O}$ emission. In general there is a good correlation between the identified molecular cores and the dark regions in the optical image, except for core E. According to the intensity distribution observed in $\mathrm{C}^{18} \mathrm{O}$, and assuming the intensity traces mass, most of the mass of the finger appears to be located in the tip of it, i.e. in core $\mathrm{C}$. The mass is apparently not symmetrically distributed along the finger but appears to be mainly located in the western part. This asymmetry is also seen in the IR image (Fig. 6), in the sense that the stellar density is much smaller in the western part of the trunk, indicating a higher extinction and therefore higher column density.

\section{Discussion}

\subsection{The molecular cores}

With the millimetre observations, we are able to estimate the excitation temperature and estimate the masses of the molecular cores. Assuming the cores are in LTE, the kinetic temperature can be derived from the $\mathrm{CO}$ spectra if it is assumed that the CO is optically thick (Rohlfs \& Wilson 1996). As the ratio between the $\mathrm{CO}$ and $\mathrm{C}^{18} \mathrm{O}$ is never above 40, this is a fair assumption since the abundance ratio is closer to 500 . Several arguments point towards the temperature derived being a lower limit: It has been assumed that the temperature is uniform within the beam and it has been assumed that the structures are resolved such that the filling factor is one. Since the sizes of the cores are comparable to the beam-width, only the spectrum obtained at the centre of a core has been used to derive the temperature to reduce the risk of having a partially filled beam.

The cores are found to be warm at $40 \mathrm{~K}, 48 \mathrm{~K}, 36 \mathrm{~K}$ and $45 \mathrm{~K}$ for core $\mathrm{N}, \mathrm{E}, \mathrm{C}$, and $\mathrm{S}$, respectively, similar to the core in the northern elephant trunks. The main uncertainties in these temperature estimates are the assumption of LTE and the implicit assumption that the beam filling factor is the same for the two sets of observations.

The temperatures found here can be compared with the temperatures found by $\mathrm{Wh} 99$ using the $\mathrm{CO}(3-2)$ transition for core $\mathrm{C}$ and $\mathrm{N}$. The temperature is, however, not explicitly given by Wh99 but counting contour levels in their CO peak temperature map gives roughly $50 \mathrm{~K}$, somewhat warmer than the temperatures we find for the core $\mathrm{N}$ and core $\mathrm{C}$. One possibility for this difference is, as already mentioned, that LTE does not apply and a more detailed analysis is necessary to obtain more accurate estimates. Also, the smaller beam for the $\mathrm{CO}(3-2)$ observations means that beam dilution is a smaller problem.

$\mathrm{CO}$ emission mainly traces the temperature in the outer part of a molecular cloud, however. In the denser inner parts where $\mathrm{C}^{18} \mathrm{O}$ and $\mathrm{CS}$ originates, the temperature can be different. The best handle we got on the temperature in the inner parts comes from Wh99. For core C, Wh99 have obtained both line emission data and continuum data from $350 \mu \mathrm{m}$ to $2000 \mu \mathrm{m}$. Using these to constrain a spectral energy distribution fit, they estimated the temperature to be $10 \mathrm{~K}$ for the dust. Since this estimate is tracing mainly temperature in the dense inner part, this temperature is more appropriate when discussing the inner part. This is in good agreement with the models by Gierens et al. (1992) which show that at least a two component model is needed to model photodissociation regions. The $\mathrm{CO}$ traces 

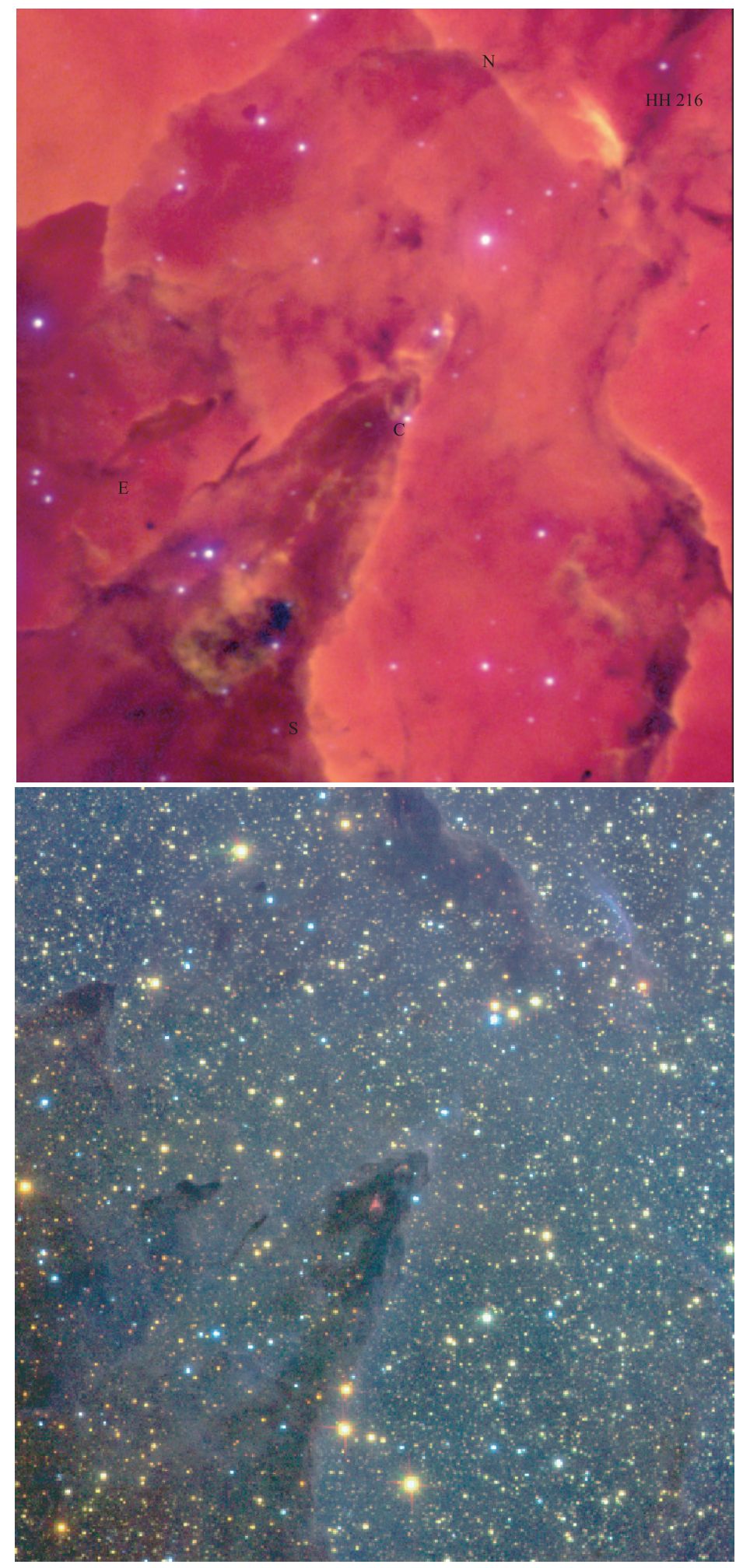

Fig. 8. Top: colour composite of the $\mathrm{H} \alpha$ (red), [S II] (green) and continuum (blue) images. The region shown is the same as is shown in Fig. 6. The intensity scale is logarithmic. The intensities for each image have been chosen to show the HH flow clearly on the H $\alpha$ background. HH objects will show up as green or yellow depending on the strength in [S II]. The cores and HH 216 are indicated with letters next to them. The HH flow is clearly seen extending along the finger and HH 216 can be seen as bright yellow. Bottom: Same region as in Fig. 6 but as a true-colour JHK image. The intensity scale is a square root stretch. Two emission nebulae are seen as very red objects associated with core C.

the warm surface, while the centre region, where the CS and $\mathrm{C}^{18} \mathrm{O}$ emission originates, can be much colder.
If we continue to assume LTE, and an inner core temperature of $10 \mathrm{~K}$, and that the $\mathrm{CS}$ and $\mathrm{C}^{18} \mathrm{O}$ emission is optically 


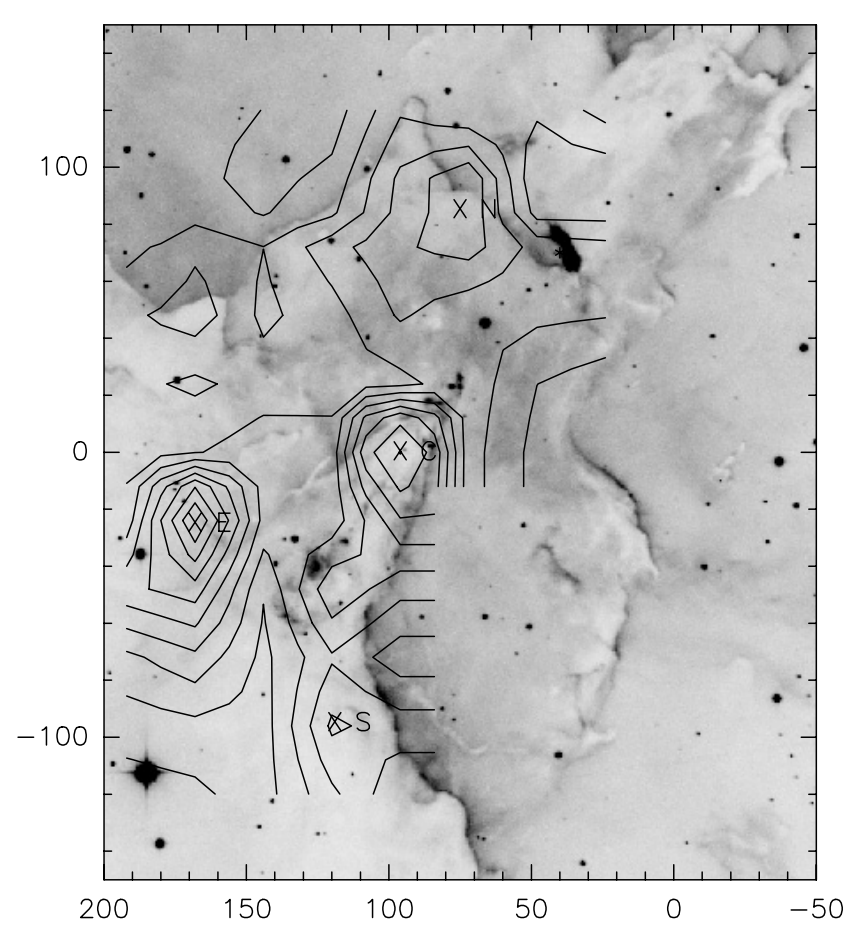

Fig. 9. A [S II] image of the regions observed at millimetre wavelengths. North is up and east is left. Overlayed as contours is the integrated $\mathrm{C}^{18} \mathrm{O}$ emission. The units on the axes are in arcseconds offset from the reference point $\left(18^{\mathrm{h}} 18^{\mathrm{m}} 52.8^{\mathrm{s}},-13^{\circ} 53^{\prime} 00^{\prime \prime}\right)$. The base of the elephant trunk complex studied by He96 with the HST is seen in the NW corner. Cores and HH 216 are marked as in Fig. 1.

thin, it is possible to estimate the masses of the cores (Rohlfs $\&$ Wilson 1996). To convert the isotope column density into a $\mathrm{H}_{2}$ column density a isotope column density conversion into $A_{V}$ using the relations by Lada et al. (1994) has been performed. This value has then been translated into $N_{\mathrm{H}_{2}}$ using the relation from Bohlin et al. (1978) The second conversion introduces the largest errors into the column density estimations. The masses derived are not very sensitive to the temperature assumed, compared to the uncertainty of $50 \%$ in the Bohlin et al. conversion.

Another independent mass estimate can be found assuming the cores are in virial equilibrium. MacLaren et al. (1988) give the virial masses for different mass profiles. The smallest virial mass is found if one assumes a $r^{-2}$ density profile. The exponent of -2 can be verified as reasonable, assuming a density distribution of $r^{-\alpha}$ and assuming the sizes of the cores are determined by the beam sizes. $\alpha$ is found to be 1.5 for core $\mathrm{N}$ and 3.0 for core $\mathrm{C}$, indicating that $\alpha=2$ is a reasonable assumption. The $\mathrm{CO}$ spectrum is (at a given position) always much broader than the optically thin spectra. Since the $\mathrm{CO}$ emission also traces low column densities, it is quite possible this broadening is due to low column density molecular material within the beam moving with slightly different velocities than the molecular material traced by $\mathrm{CS}$ and $\mathrm{C}^{18} \mathrm{O}$. We therefore use the $\mathrm{CS}$ and $\mathrm{C}^{18} \mathrm{O}$ spectra to estimate virial masses.

Table 2 lists the estimates of masses using the different methods and compares with the mass estimates from Wh99. For the virial mass estimates an $r^{-2}$ density profile was
Table 2. Column (a): core mass estimate assuming LTE and using $\mathrm{C}^{18} \mathrm{O}$. Column (b): as (a) but using CS. Column (c): the mass found by Wh99. Column (d): the virial mass using the line-widths found for $\mathrm{C}^{18} \mathrm{O}$. Column (e) as (d) but using the line-widths found for CS. All masses are in solar masses.

\begin{tabular}{llcccc}
\hline \hline Core & $\begin{array}{l}M_{\mathrm{C}^{18} \mathrm{O}} \\
(a)\end{array}$ & $\begin{array}{c}M_{\mathrm{CS}} \\
(b)\end{array}$ & $\begin{array}{c}\mathrm{Wh} 99 \\
(c)\end{array}$ & $\begin{array}{c}M_{\mathrm{vir}}\left(\mathrm{C}^{18} \mathrm{O}\right) \\
(d)\end{array}$ & $\begin{array}{c}M_{\mathrm{vir}}(\mathrm{CS}) \\
(e)\end{array}$ \\
\hline $\mathrm{N}$ & 85 & 60 & 35 & 165 & 185 \\
$\mathrm{C}$ & 95 & 65 & 80 & 110 & 140 \\
$\mathrm{E}$ & 87 & 100 & - & 125 & 145 \\
\hline
\end{tabular}

assumed. Considering the assumptions made, the results from the two different isotopes agree rather well for core E. The main reason for the large difference in mass between this study and Wh99 is due to a probable overestimate of the core radius due to the larger beam-width in the $2-1$ transition than the 3-2 transition used by Wh99. The overestimate can be seen fairly easy in Figs. 6 and 9 by comparing the size of the contours with the actual size of core $\mathrm{N}$, for example. Also, from the near-infrared data it is apparent that the cores are not spherical.

The agreement is quite good for core $\mathrm{C}$, although the conversion factors used by Wh99 should result in slightly smaller mass estimates than in this study. Due to our large beam compared to the sizes of the cores, most of the mass estimates are probably larger than would be found with a smaller beam.

It is clear that the virial masses are much higher than the mass estimates from LTE, except for core E. This discrepancy is not likely to be due to wider lines caused by the UV flux, since the CS emission originates deep in the core. It appears that, besides core $\mathrm{E}$, the cores are probably not virialised at the present time. The better correspondence for core E could be due to its larger size and therefore a smaller dependency on the large beam. Also, at low temperatures, $\mathrm{CO}$ and $\mathrm{C}^{18} \mathrm{O}$ molecules might freeze out and the $\mathrm{C}^{18} \mathrm{O}$ emission will then not trace all the mass, reducing some of the discrepancy between the column density and the virial mass estimates.

\subsection{A possible $\mathrm{HH}$ jet and a driving source}

The string of emission features seen in [S II] in Fig. 9 is also seen in $\mathrm{H} \alpha$ emission. Figure 7 shows the region just around the finger and includes possible $\mathrm{HH}$ objects and also $\mathrm{HH} 216$. A comparison between the [S II], $\mathrm{H} \alpha$, red continuum and $K_{\mathrm{S}}$ band images can be seen in Fig. 6. Figure 8 shows a colour composite of the $\mathrm{H} \alpha$ (red), [S II] (green) and the continuum (blue) images.

Images in $\mathrm{H} \alpha$, [S II] and red continuum are very powerful when searching for $\mathrm{HH}$ objects. Since an $\mathrm{HH}$ object is strong in lines and not in continuum, it will show up in these frames and not in the continuum (see e.g. Reipurth 1989). While this is a powerful technique in dark clouds, it works less well in H II regions, where photoionised gas also shows up in $\mathrm{H} \alpha$ and [S II]. In such cases, additional information is required, either from the morphology or the kinematics of the objects.

The morphology and velocity structure seen in the optical and near-infrared suggest that HH216 can be extended from 
being an isolated $\mathrm{HH}$ object to be part of a large $\mathrm{HH}$ flow on the following grounds. The string of narrow-band emission follows a straight line that, if extended, passes through $\mathrm{HH} 216$. $\mathrm{HH} 216$ and the string of emission knots are moving in radial directly opposite directions with the same absolute speed of $150 \mathrm{~km} \mathrm{~s}^{-1}$, consistent with HH flow velocities. Using an image from July 1985 in combination with the 1999 data, a test for proper motion has been performed. No proper motion was found over this 14 years baseline, setting an upper limit of $200 \mathrm{~km} \mathrm{~s}^{-1}$, still consistent with a HH flow inclined at least $36^{\circ}$ with respect to the plane of the sky.

From HH 216 to the putative counter bow shock, the total apparent length of the flow is $1.63 \mathrm{pc}$. Since no proper motion was found, we can only place a lower limit on the dynamical age of the flow under the assumption of no deceleration of the flow. The dynamical time is given by $t_{\mathrm{dyn}}=L / V$, where $L$ is the projected length of the flow and $V$ is the tangential velocity. With the numbers quoted above, the lower limit is $t_{\mathrm{dyn}} \sim 10^{4} \mathrm{yr}$.

HH 216 shows up weakly in the red continuum and in the near-infrared. This continuum emission has already been noticed in the optical by Meaburn \& Walsh (1984). HH 216 was also observed in the continuum at $8.7 \mathrm{GHz}$ by Wh99. The weak continuum of $\mathrm{HH}$ objects are usually ascribed to collisionally enhanced two-photon decay in neutral atomic hydrogen (e.g. Brugel et al. 1982). Since HH 216 is presumably also irradiated by the UV flux from the $\mathrm{O}$ and $\mathrm{B}$ stars, this could also create some continuum flux. Given that our optical continuum observations were not flux calibrated, the relative strength between the $8.7 \mathrm{GHz}$ continuum emission and the optical continuum cannot be estimated.

Having an HH flow, a driving source still needs to be identified. Assuming the terminating shock of the redshifted part of the flow is $\mathrm{HH} 216$ and the terminating shock of the counter flow is located at $(135,-60)$, the driving source should, for symmetry reasons, be located within core $\mathrm{C}$. This is where we see a complex extended nebulosity in the infrared images. It is located very close to the symmetry axis of the HH flow, as seen in Fig. 7, strongly suggesting that this region may harbour the HH216 driving source. The northern tip of this nebulosity is almost starlike, while the rest is in the form of a small cometary nebula, and at present, based on broad-band images alone, it is not possible to tell whether this extended nebulosity is reflected continuum or molecular hydrogen emission. Equally, from the present data alone, we are unable to determine whether the driving source is coincident with the starlike object or is more deeply embedded, invisible at these wavelengths. Thermal-infrared imaging may settle that question. Nevertheless, the apparent superposition of the HH flow, nebulosity, and molecular core strongly suggest that Core $\mathrm{C}$ is the site of recent star formation.

\section{Conclusions}

The base of the elephant trunks in M 16 has been observed at millimetre, optical, and near-infrared wavelengths. The millimetre maps show several molecular cores and an elephant trunk. Two of the cores have previously been observed in other isotopes. The elephant trunk shows almost no sign of a velocity gradient, indicating that the trunk is probably not formed by a Rayleigh-Taylor instability; this agrees with the findings of P98 and Wh99 for the trunks further to the north. The cores appear to be externally heated with surface temperatures between 36 and $48 \mathrm{~K}$ and are cold in the central regions ( $\sim 10 \mathrm{~K})$. This supports the simple expectation that UV radiation from the nearby OB cluster is heating the cloud. Due to the relatively large beam in our observations, the filling factor might be smaller than one and the temperatures found above should be regarded as lower limits.

By using the $\mathrm{CS}$ and $\mathrm{C}^{18} \mathrm{O}$ spectra, masses are derived for the molecular cores, resulting in values between $60 M_{\odot}$ and $100 M_{\odot}$. By comparing with the masses found from the velocity dispersion it does not appear that the molecular cores are virialised, the virial masses are consistently higher than the LTE derived masses except perhaps for core E. An alternative explanation could be that due to the low temperatures and high densities in the centre of cores, the $\mathrm{C}^{18} \mathrm{O}$ molecules have been frozen out and are not tracing mass. It is somewhat curious since this is the core that has apparently not yet been severely affected by the UV flux. The rest of the discrepancy could be due to the LTE assumption in both our study and the study of Wh99, and to some extent the different beam sizes. The mass derived in this study for core $\mathrm{N}$ is much larger than estimated by Wh99, presumably due to the LTE assumption and uncertainties in core radii. For core $\mathrm{C}$ the agreement with Wh99 is quite good.

We have identified what appears to be a counterpart to $\mathrm{HH} 216$. Whereas HH 216 is redshifted with a radial velocity of about $+150 \mathrm{~km} \mathrm{~s}^{-1}$, the new emission-line object is blue-shifted with a radial velocity of about $-150 \mathrm{~km} \mathrm{~s}^{-1}$. Several other optical or infrared knots are found on a line between these two objects. Mid-way between these two objects we find a small near-infrared extended reflection nebula, which is likely to be a signpost of the embedded driving source of an extended system of HH objects, which we suggest calling the HH 216 flow. Thus, the observations presented here indicate that star formation is taking place within core $\mathrm{C}$.

Acknowledgements. MA acknowledges support from the Julie Marie Vinter Hansen foundation for travel support. We thank Gilles Duvert and Henrik Beuther for support with software and the referee Dr. A. Gibb for useful comments and suggestions. We are grateful to Lise Christensen, AIP for obtaining some of the optical images.

\section{References}

Belikov, A. N., Kharchenko, N. V., Piskunov, A. E., \& Schilbach, E. 2000, A\&A, 358, 886

Bohlin, R. C., Savage, B. D., \& Drake, J. F. 1978, ApJ, 224, 132

Brugel, E. W., Seab, C. G., \& Shull, J. M. 1982, ApJ, 262, L35

Cernicharo, J. 1991, in The physics of star formation and early stellar evolution, ed. C. Lada, \& N. Kylafis, NATO, ASI Ser. C., 342 (Dordrecht: Kluwer)

Currie, D., Kissell, K., Shaya, E., et al. 1996, ESO Messenger, 86, 31 Gierens, K. M., Stutzki, J., \& Winnewisser, G. 1992, A\&A, 259, 271

Hester, J. J., Scowen, P. A., Sankrit, R., et al. 1996, AJ, 111, 2349 [He96] 
Hillenbrand, L. A., Massey, P., Strom, S. E., et al. 1993, AJ, 106, 1906 [Hi93]

Krautter, J., Reipurth, B., \& Eichendorf, W. 1984, A\&A, 133, 169

Lada, C. J., Lada, E. A., Clemens, D. P., \& Bally, J. 1994, 429, 694

MacLaren, I., Richardson, K. M., \& Wolfendale, A. W. 1988, ApJ, 333,821

McCaughrean, M. J., Rayner, J. T., \& Zinnecker, H. 1994, ApJ, 436, L189

McCaughrean, M. J., \& Andersen, M. 2002, A\&A, 389, 513

Meaburn, J., \& White, N. J. 1982, MNRAS, 199, 121

Meaburn, J., \& Walsh, J. R. 1984, A\&A, 138, 36

Pilbratt, G. L., Altieri, B., Blommaert, J. A. D. L., et al. 1998, A\&A, 333, L9
Pound, M. P. 1998, ApJ, 493, L113 [P98]

Reipurth, B. 1989, A\&A, 220, 249

Rohlfs, K., \& Wilson, T. L. 1996, Tools of radio astronomy (New York: Springer Verlag), 2nd ed

Sugitani, K., Tamura, M., Nakajima, Y., Nagashima, C., et al. 2002, ApJ, 565, L25

Thompson, R. I., Smith, B. A., \& Hester, J. J. 2002, ApJ, 570, 749

Walsh, J. R., \& White, N. J. 1982, MNRAS, 199, 9

White, G. J., Nelson, R. P., Holland, W. S., et al. 1999, A\&A, 342, 233 [Wh99]

de Winter, D., Kouis, C., Thé, P. S., et al. 1997, A\&AS, 121, 223 